\title{
Android Application to Manage the Rescue Interventions During Power Outages : Design and Implementation
}

\author{
H. Hajji, M. Bouzi \\ Laboratory of Mechanical Engineering, \\ Industrial Management and Innovation \\ University Hassan 1, Settat, Morocco.
}

\author{
K. Rhofir \\ Laboratory of Informatics, \\ Systems, Electric, Network and telecom \\ National School of Applied Sciences, \\ Khouribga, Morocco.
}

\begin{abstract}
The Decision-making in the field of management of electricity distribution networks faces uncertain and multiobjective challenges. For this type of problem, decision-making often requires the optimization of functions with several objectives that are generally contradictory.

In addition, the accelerated change in the environment (imposed by the increasing customers' demands, the industry 4.0, the evolution of technology and competition) shortens significantly the decision-making deadlines while making this task more difficult for distribution network operators. New technologies and mainly smart technologies are therefore becoming a necessity.

In this work, in order to meet this need, we have implemented a decision application tool based on Java Android technology and artificial intelligence using the fuzzy logic.

The main challenge of this mobile application is to offer a framework to help managers optimize their rescue interventions and much better the customer expectations in case of the electricity distribution network breakdown.
\end{abstract}

Keywords - Mobile application, Android, Fuzzy logic, Decision making, electricity breakdown.

\section{INTRODUCTION}

In today's competitive business environment, companies face challenges in dealing with the issues of fast decisionmaking for increased productivity. Many companies are leading a transformation towards a 4th generation industrial revolution (Industry 4.0) by integrating and / or embedding intelligent analytical tools in their systems.

To contribute to this transformation, the project is to create a mobile application using smartphones. The tool named "eSecours" is able to help managers decide in real time what to do to rescue the power distribution network in case of failure.

The expected results of such application are:

- Increasing productivity by reducing decision-making time and the effectiveness of interventions.

- Improving services to customers. In particular, the quality and the intervention deadlines of the field teams.

- Improving working conditions through job enrichment and digitization.

- Improving data traceability by digitizing anomalies and reducing documents to archive.

The installation of this application will be very easy and will not require investment or special logistics since it will use the company's computer network and the existing smartphones and tablets (which are equipped the organization's managers).

In this paper, fuzzy logic is introduced to take account of the weaknesses displayed by the current practices and decisions, in terms of their responsiveness in an imprecise environment and the variables are not homogeneous. Specifically, with fuzzy logic we are able to obtain a single value (output) from the imprecise data (input).

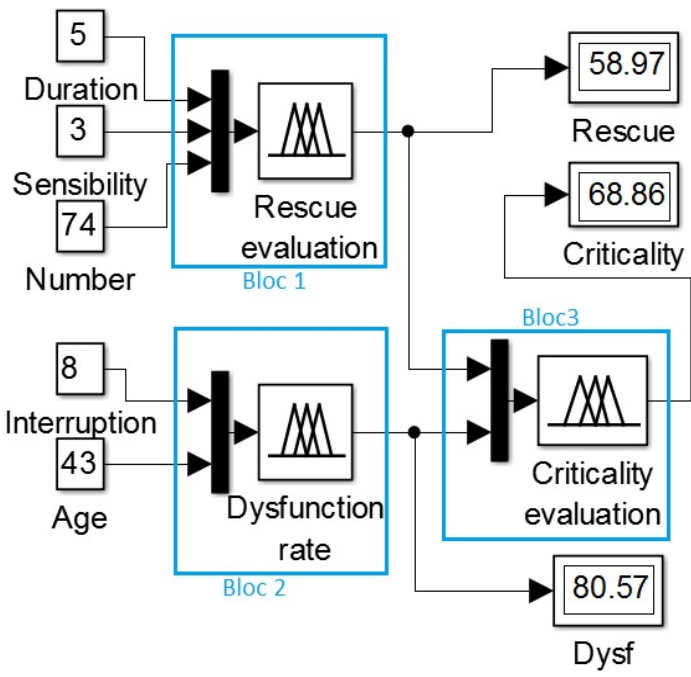

Figure 1: Architecture of the system.

The proposed model is divided on three parties that based on Mamdani fuzzy inference [1] [2] [3]. The first bloc uses as input variables: the duration of the interruption, the customers' sensitivity and the number of clients to restore. The output is the net value of the emergency level.

The second bloc uses as input variables: the age and the interruption rate of the network. The output is the dysfunction rate of the electrical network.

These two outputs variables are re-injected in the third bloc as input variables in order to get a level of criticality [4]. It allows us to determine the severity level of interruption, if it is acceptable or not, in order to better control risks and manage priorities. Once complete analysis, we will have all the information necessary to decide the type of rescue to be made.

This paper is organized as follows: section II, gives a description of the proposed mobile application based on Java Android technology. Section III presents the system design. 
Section IV develop the system implementation, using Android studio platform. Section V presents the evaluation and results. The conclusions are given in section VI.

\section{SYSTEM DESCRIPTION}

To realize our application, we chose to modulate it in order to be able to better manage its development as well as its maintenance. It will be split into Three Tier Architectures: [5]

- Database layer (DB) that allows storage and access to data.

- Application layer that allows the processing of data; by implementing the application-specific features to describe the operations that the application performs on the data based on user requests.

- Presentation layer whose function is to create the user interface (display, rendering on the workstation, dialogue with the user ...).

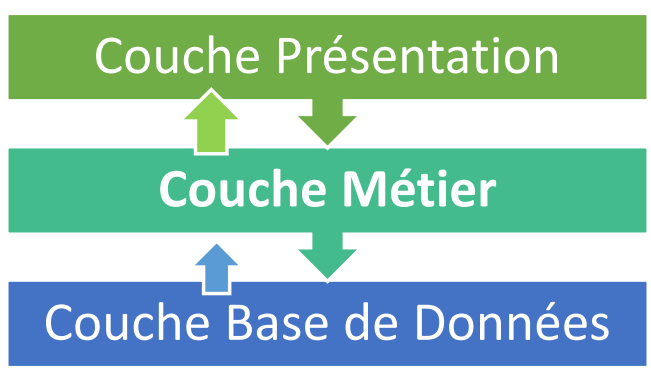

Figure 2: Implementation in Three-Tier Architecture

\section{SYSTEM DESIGN}

A. Introduction

The general diagram of the Generators mobilization logic is shown in Fig.3:

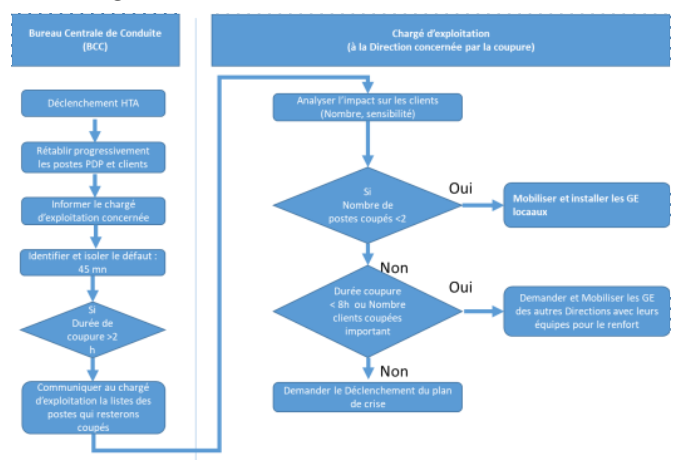

Figure 3: Generator mobilization logic diagram

When an outage happened, we start by:

- Diagnosis: of the incident based on the information transmitted by the fault detectors placed along the HTA loop.

- Localization of the defect: execution of the maneuver sequences on the remote controlled works (the closest to the defect) then by the operators, on site, in order to refine the localization.

- Isolation of the fault and resumption of service: Truncation, resumption by the available emergency departures and return of the voltage.
- Management of the intervention teams: these teams, equipped with tablets, receive the maneuvers to be performed, the maneuvering sheets to be produced and the plans or cards required for the intervention.

- Customer information: communicating interrupt information from the network topology. This information concerns the affected customers and the estimate of the duration of the cut depending on the maneuvers to be performed. [6]

The design idea of our software "eSecours" based on Android is described as follows (Fig.4):

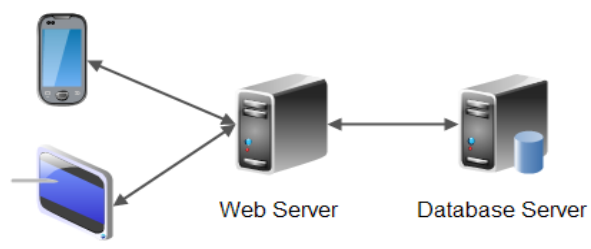

Figure 4: General Design

First through authentication, the Android user send a request to the server.

Then the server will parse the requested parameters.

If it is a request from the central control office, then the user will select the list of failed stations, possibly update their respective data (age, interruption rate, average sensitivity and number of customers) and record the duration of the outage.

If the request is from one of the local manager then the server will send the data of the cut stations to the specified local manager. Witch, after having made a field visit and possibly modify the data of each station, he validates the request on his smartphone.

Then the server will operate the local database and return the result to the user. It proposes a level of assistance to be provided to the cut stations and evaluates the criticality of the incident to allow prioritizing the interventions.

The Android-side of the system is developed on Android Studio platform, and the programming language is Java.

B. Hardware environment

The hardware environment of our application takes full advantage of the revolution of our use of new technologies. We are talking about smartphones and tablets that have transformed our way of life in just a few years.

To implement our software, we used the following material:

- Smartphones.

- Tablets.

- A servers.

- A sim card 3G/4G.

\section{Software environment}

In general, the development environment consists of a set of tools that increases the productivity and efficiency of the final rendering. It includes:

a) Programming languages

Java Android: For the development of user interfaces. It is the official IDE for the Android application development. [7]

php: For the intranet development of the enterprise website. 


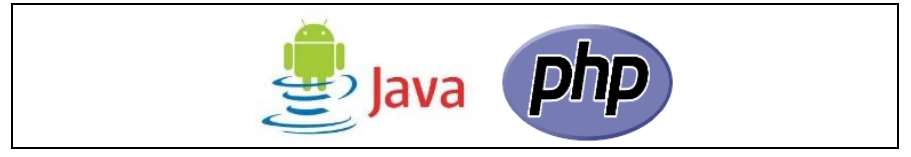

b) Programming tools

To take full advantage of the features of the smartphone used, we have opted for Cordova/ PhoneGap. It allows local file backup, use of the camera, GPS and sensory sensor or access to contacts.

For the user interfaces, we have used Adobe XD and Android studio to view pages and make them automatically adapt to the tool used.

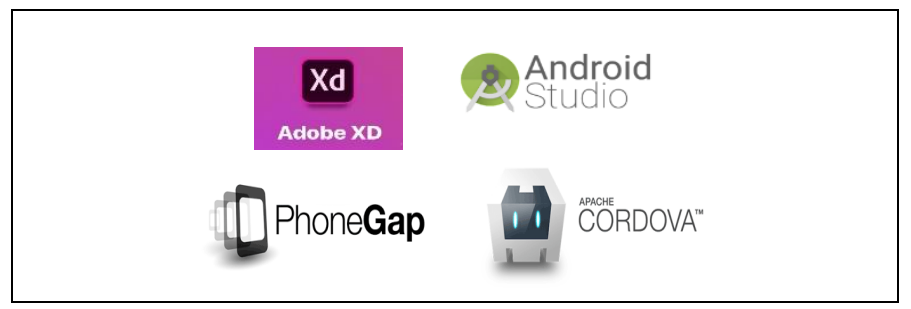

\section{c) Editor (IDE)}

For the edition of our code, we used the Open Source Notepad ++ (or Visual Studio Code). For its simplicity and completeness for the entire cycle of editing and debugging.

\section{d) Library}

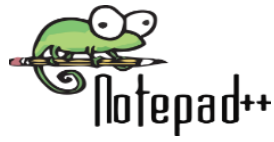

The library used in the development of this application is JFuzzyLogic.

This is the most complete open source Java library, using the standard language Fuzzy Control Language (FCL). It is a language that allows a complete and functional implementation of a fuzzy inference system (FIS). It provides a programming interface and a plugin for easily writing and testing the code of applications based on fuzzy logic. It has been standardized by IEC 61131-7. [8]

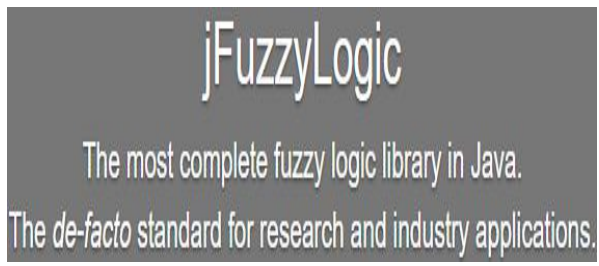

\section{e) Database Management}

phpMyAdmin: Allows data management with a graphical interface. Support for most MySQL features. Browse and add databases, tables, fields and indexes. Create copy, rename and modify databases, tables, fields and indexes. Server maintenance, databases and tables, proposals on server configuration. Manage MySQL users and privileges. Manage stored procedures and triggers. Data import from CSV and SQL. Export data to different formats: CSV, SQL, XML, PDF, ISO / IEC 26300 - Open Document Spread Sheet, Word, Excel, Latex and others. [9]
MySQL: it is a database system used on database server. With PHP, we can connect to and manipulate databases.
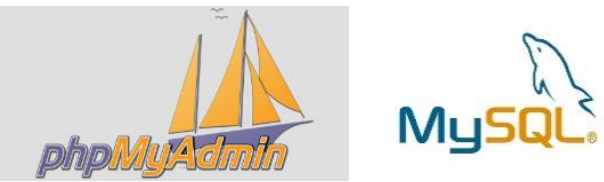

\section{f) WebServer Management}

Apache: Apache Web Server is designed to create web servers that have the ability to host one or more HTTP-based websites. It's a remote computer that serves files to requesting clients.

\section{IV.SYSTEM IMPLEMENTATION}

The figure above represents the logo we chose for our application.

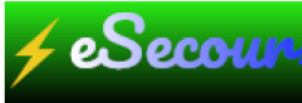

When launching the application the user sees the following flash interface:

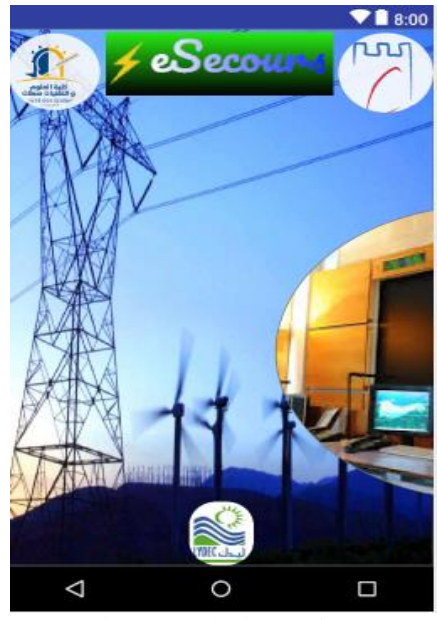

Figure 5: Flash interface

A. Authentication panel

We start by entering the user's name and the password.

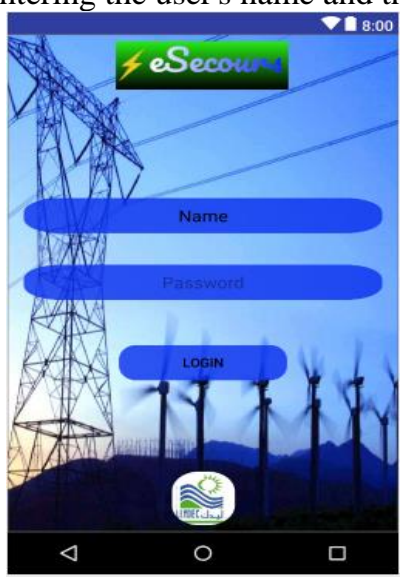

Figure 6: Authentication interface

Then, we validate by pressing login. Two scenarios can arise: it depends on whether you are the operator Headquarters (the central control office) or a local manager among the seven prefectural directorates. 


\section{B. Edition Data Interface}

First situation: the request is from the central control office, then the user will select the list of failed stations, possibly update their respective data (age, interruption rate, average sensitivity and number of customers) and record the duration of the outage.

Second situation: the request is from one of the local manager then the server will send the data of the cut stations to the specified local manager. Witch, after having made a field visit and possibly modify the data of each station, he validates the request on his smartphone.

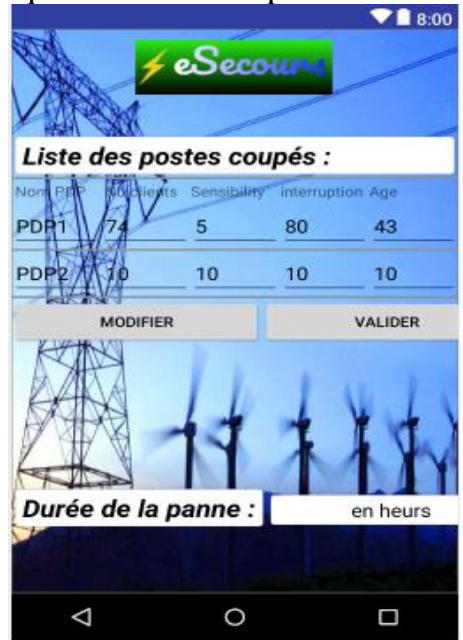

Figure 7: Edite Data interface

\section{Decision panel}

Then the server operates the local database and return the result to the user. It proposes a level of assistance to be provided to the cut stations and evaluates the criticality of the incident to allow prioritizing the interventions.

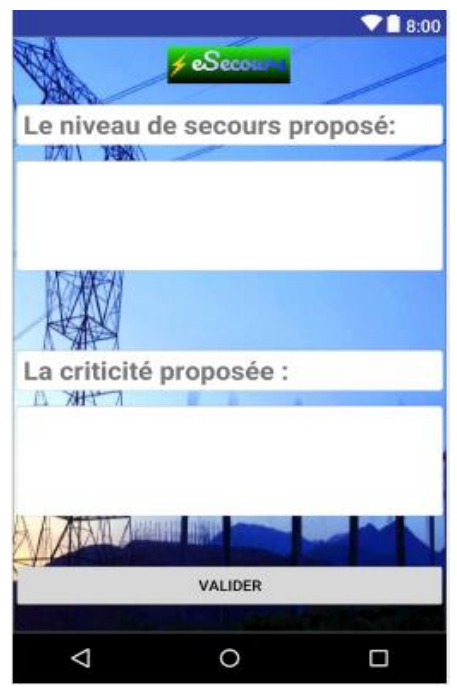

Figure 8: Decision interface

\section{Tasks and estimations}

Depending on the criticality values, actions must be taken to solve the problem of power failure. Its actions are sent to the main server and processed according to their degree.
We have also proposed a function that calculates the difference between the real solution and that proposed by our application. The result is also sent to the server for backup.

These two elements feed our knowledge base for better decision-making in the future.

\section{EVALUATION AND RESULTS}

This software runs on a smartphone (galaxy j7 pro), and the version of Android is 7.0

The network environment is WIFI or 3G.

The login time is less than 2 seconds.

The switch between UI runs smoothly.

Moreover, no database or message disorder occurred in our software communication module.

The classification of function in this application is clear and easy to deploy.

The output data are compatible with the users' expectations.

\section{CONCLUSION}

In the general context of competition between the managers of the electricity distribution network accentuated by the liberalization of the electricity market at the global level, the need for the optimization of the practices deployed to satisfy private and constitutional customers has become essential. Innovation in its services to customers is becoming increasingly important as their requirements increase.

The main objective of the research works carried out in this article concerns the decision-making problem of management of outages on the electrical distribution network.

The purpose of this work was to contribute to the optimization of systems in general by using techniques related to artificial intelligence. More precisely, fuzzy logic. The choice of this method was not made arbitrarily, but rather because of the nature of our decision-making system. The latter has non-linear characteristics.

For this, we exposed the current procedure of fault management where we were able to visualize in a sequential and logical way the actions carried out, by the operators, during a triggering on an electrical network. We are facing human's decisions, taken to rescue the needy customers by generators.

In addition, we have detailed our mobile application developed specifically for the project. This application is based on fuzzy logic to provide operators with a decisionmaking tool.

The results are verified and then tested. They are all concurrent and meet the expectations of users. This shows the interest of our application as an effective tool of decision support for the managers of the electrical distribution network.

On perspective, the application can be used as a pedagogical complement in the field of fuzzy logic applications.

We can also try to experiment the presented approach. The implementation of this method to real cases of power outages will compare the different assumptions expressed. 


\section{REFERENCES}

(1) F.Dernoncourt, «Introduction à la logique floue,» 84 2011. [En ligne]. Available: http://franckdernoncourt.developpez.com/tutoriels/algo/introduction-logiquefloue/.

(2) G. \&. F. O. Buyukozkan, «A fuzzy logic based decision making approach for new product development,» International Journal of Production Economics, vol. 90, pp. 27-45, 2004.

(3) G. Gheorghe, «Using K-Means Clustering Method in Determination of the Energy Losses Levels from Electric Distribution_ ystems,» chez 12th WSEAS International Conference, Faro, Portugal, 2010.

(4) Wikipedia, «Criticality index,» 1102 2018. [En ligne]. Available: https://en.wikipedia.org/wiki/Criticality_index. [Accès le 2018].

(5) «Multitier architecture,» 04 Sept 2018. [En ligne]. Available: https://en.wikipedia.org/wiki/Multitier_architecture. [Accès le 2018].

(6) SDA, «Le système de télé conduite du réseau de distribution électricité HTA,» [En ligne]. Available: www.sda.dz/Media/upload/file/Teleconduite_SDA.pdf.

(7) «Meet Android Studio,» 24 Sept 2018. [En ligne]. Available: https://developer.android.com/studio/intro/.

(8) wikipedia, «Fuzzy Control Language,»02 Aout 2015. [En ligne]. Available: https://en.wikipedia.org/wiki/Fuzzy_Control_Language.

(9) «phpMyAdmin,» 14 Février 2015. [En ligne]. Available: http://www.phpsources.org/ressources-phpMyAdmin-php691.htm. [Accès le 2018]. 